\title{
Avaliação da autofluorescência do fundo de olho nas distrofias de retina com o aparelho Heidelberg Retina Angiograph2
}

\author{
Evaluation of fundus autofluorescence in hereditary retinal \\ diseases using Heidelberg Retina Angiograph2
}

Monique Côco ${ }^{1}$

Natalia Tamie Baba ${ }^{2}$

Juliana Maria Ferraz Sallum ${ }^{3}$

\section{RESUMO}

Objetivos: Definir características do exame de autofluorescência, verificando sua utilidade no diagnóstico e acompanhamento de distrofias retinianas. Métodos: Participaram do estudo, 28 pacientes, adultos, divididos igualmente em quatro grupos com diagnósticos de doença de Stargardt, distrofia de Cones, retinose pigmentar e voluntários saudáveis para estabelecimento do padrão de normalidade. Em média foram obtidas nove imagens com o filtro para angiofluoresceinografia para a formação da imagem autofluorescente no Heidelberg Retina Angiograph2. As imagens de cada grupo de pacientes foram analisadas para verificar características comuns. Resultados: As imagens fundoscópicas autofluorescentes dos voluntários do grupo controle mostraram área foveal hipoautofluorescente em relação à retina do pólo posterior. As imagens dos portadores de doença de Stargardt, em geral, apresentaram lesão hipoautofluorescente, correspondendo à área macular. As principais alterações da autofluorescência em pacientes com distrofia de cones foram hipoautofluorescência macular com halo hiperautofluorescente. Nos portadores de retinose pigmentar, foram encontrados pigmentos periféricos causando hipoautofluorescência. Na região macular, hipoautofluorescência ou apenas desorganização do pigmento. Conclusão: $\mathrm{O}$ estudo mostrou a existência de padrões de autofluorescência de fundo nas distrofias de retina que permitem o diagnóstico e melhor interpretação da fisiopatogenia destas doenças.

Descritores: Doenças retinianas; Lipofuscina; Epitélio pigmentado ocular; Oftalmopatias hereditárias; Angiofluoresceinografia

\section{INTRODUCÃO}

A autofluorescência é uma propriedade que as células retinianas apresentam pela presença de lipofuscina. A lipofuscina deriva principalmente de resíduos químicos modificados como resultado de uma digestão/metabolização incompleta dos segmentos externos dos fotorreceptores ${ }^{(1)}$.

O segmento externo dos fotorreceptores contém proteínas fotossensíveis chamadas opsinas.

A absorção de luz pela opsina induz a isomerização do 11-cis-retinal em all-trans-retinal e a conseqüente re-isomerização do all-trans-retinol em 11cis-retinol, através de um processo enzimático chamado ciclo visual ${ }^{(2)}$. A fototransdução ocorre no segmento externo dos fotorreceptores. A regeneração de 11-cis-retinol, proveniente do ciclo visual, ocorre nas células do 
epitélio pigmentado da retina (EPR), camada celular adjacente a dos fotorreceptores. Outra função do EPR é realizar a fagocitose dos resíduos químicos provenientes da metabolização incompleta pelo segmento externo dos fotorreceptores. A conseqüência desse processo é o acúmulo gradual de lipofuscina $^{(3-4)}$.

O principal componente fluorescente da lipofuscina é o A2E, derivado do subproduto A2-PE do ciclo visual, sintetizado da reação do all-trans-retinal com phosphatidylethanolamine (PE), para manter a concentração de retinol ${ }^{(3)}$ (Figura 1).

$\mathrm{O}$ gene $A B C A 4$ codifica a proteína $A B C R$, localizada no segmento externo dos fotorreceptores. Essa proteína está envolvida no transporte ativo de all-trans-retinal e phosphatidylethanolamine pela membrana dos discos intercalares dos fotorreceptores, auxiliando a eliminação desses componentes $^{(5)}$ (Figura 2).

As mutações do gene $A B C A 4$ causam uma forma autossômica recessiva de degeneração retiniana.

Estudos realizados em camundongos mostraram que a perda ou redução da função da proteína $A B C R$ causa elevação de phosphatidylethanolamine no segmento externo dos cones e no EPR, deposição de A2E e conseqüente acúmulo de lipofuscina no EPR, seguido de lenta degeneração dos fotorreceptores ${ }^{(6)}$.

$\mathrm{O}$ aumento anormal de A2E predispõe a disfunção e/ou morte do EPR seguido de perda dos fotorreceptores e diminuição da visão(6).

Estudos mostram que há correspondência entre áreas de

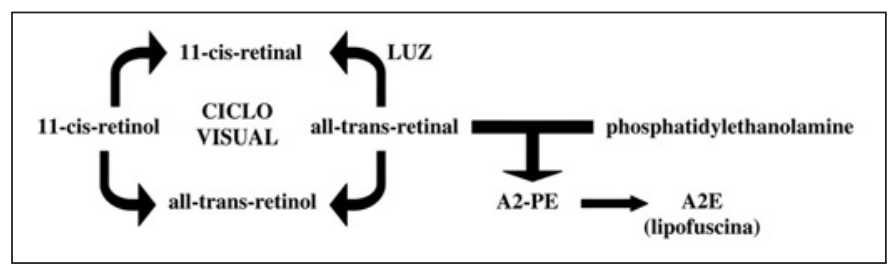

Figura 1 - Ciclo visual. All-trans-retinal reage com phosphatidylethanolamine formando A2-PE, subproduto do fluoróforo A2E.

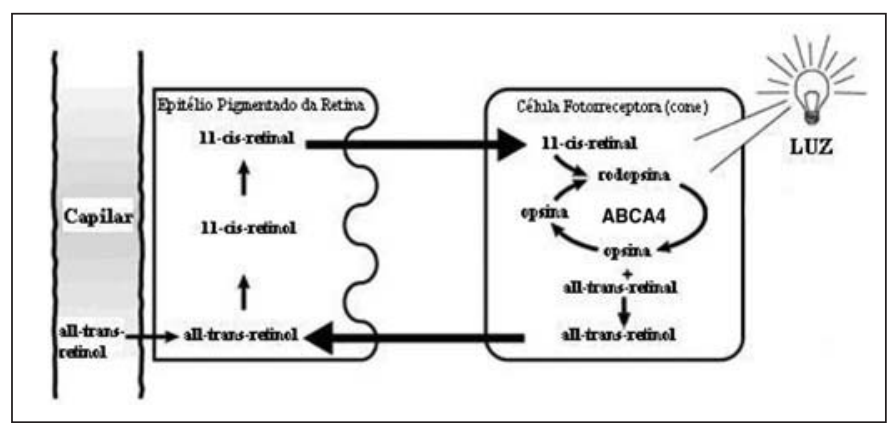

Figura 2 - Ciclo visual. Retinol chega ao epitélio pigmentado da retina (EPR) pela via circulatória, sofre hidrólise formando 11-cis-retinol. Este 11-cis-retinol por oxidação no fotorreceptor se transforma em 11-cisretinal, que junto com a opsina formam a reação de fototransdução. A absorção de luz causa isomerização de 11-cis-retinal em all-transretinal. O ABCA4 media o transportador destas substâncias através das membranas dos discos intercalares dos fotorreceptores. Depois disso, all-trans-retinalse converte em all-trans-retinolque é transportado para o EPR, completando o ciclo visual. autofluorescência diminuídas e áreas de perda de células fotorreceptoras, sugerindo que a autofluorescência seja um bom marcador para a integridade do EPR, podendo ser útil para investigar a evolução das distrofias da retina ${ }^{(1,7-8)}$.

As distrofias retinianas têm caráter hereditário e causam perda progressiva da função das células fotorreceptoras e diversos sintomas que variam conforme o tipo de lesão. Imagens fundoscópicas são usadas para diagnosticar e documentar essas degenerações ${ }^{(7,9-10)}$.

O Heidelberg Retina Angiograph2 é um sistema de laser confocal para angiografia digital. Utiliza técnica não invasiva para adquirir imagens de uma determinada área da retina ou coróide com alta precisão e velocidade, permitindo análise de progressão das doenças. Dentre vários padrões fotográficos disponíveis neste aparelho foi possível fazer fotos em padrão para examinar a autofluorescência e analisar áreas com características de hiper e hipoautofluorescência.

O objetivo deste estudo é definir as características do exame de autofluorescência do fundo de olho e verificar se este exame será útil no diagnóstico dos diversos tipos de distrofias retinianas.

\section{MÉTODOS}

Este estudo prospectivo, contou com a participação de 28 voluntários divididos em 4 grupos:

Grupo I: 7 voluntários saudáveis para controle e comparação dos exames ( 3 homens e 4 mulheres), com idades variando entre 20 a 49 anos (média de 29,84 \pm 11,34);

Grupo II: 7 Portadores de doença de Stargardt (1 homem e 6 mulheres), com idades variando entre 37 a 54 anos (média de 42,05 \pm 9,15);

Grupo III: 7 Portadores de distrofia de cones (2 homens e 5 mulheres), com idades variando entre 14 e 54 anos (média de $34,78 \pm 15,29)$;

Grupo IV: 7 Portadores de retinose pigmentar ( 5 homens e 2 mulheres), com idades variando entre 34 a 49 anos (média de $40,97 \pm 6,04)$.

$\mathrm{O}$ aparelho utilizado para fotografar e armazenar as imagens do fundo de olho foi o Heidelberg Retina Angiograph2, HRA2 (Heidelberg Engineering, Germany). Uma média de nove imagens com o filtro da angiofluoresceinografia (FA) foi obtida para a formação das imagens autofluorescentes. $\mathrm{O}$ exame foi realizado em ambos os olhos, sendo necessário a dilatação pupilar. Através das imagens obtidas, três examinadores analisaram as características como intensidade da fluorescência ou refletividade e padrão de distribuição das lesões encontradas em portadores de diferentes tipos de distrofias retinianas.

Posteriormente, essas imagens foram comparadas dentro de cada grupo para se obter características comuns de uma determinada distrofia.

Foram considerados como critérios de inclusão, para o grupo de pacientes com distrofias, o diagnóstico clínico das 
respectivas distrofias de retina, ausência de doenças sistêmicas, concessão do termo de consentimento livre e esclarecido, acuidade visual com melhor correção óptica em um ou ambos os olhos, melhor que 1,3 logMAR (20/400 Snellen), e para o grupo de normalidade, acuidade visual 0,0 logMAR (20/20 Snellen).

O estudo foi aprovado pelo Comitê de Ética em Pesquisa da Universidade Federal de São Paulo/Hospital São Paulo (CEP 1653/06).

\section{RESULTADOS}

A acuidade visual tornou-se critério de inclusão para facilitar a fixação da mira pelo paciente e assim obter melhores
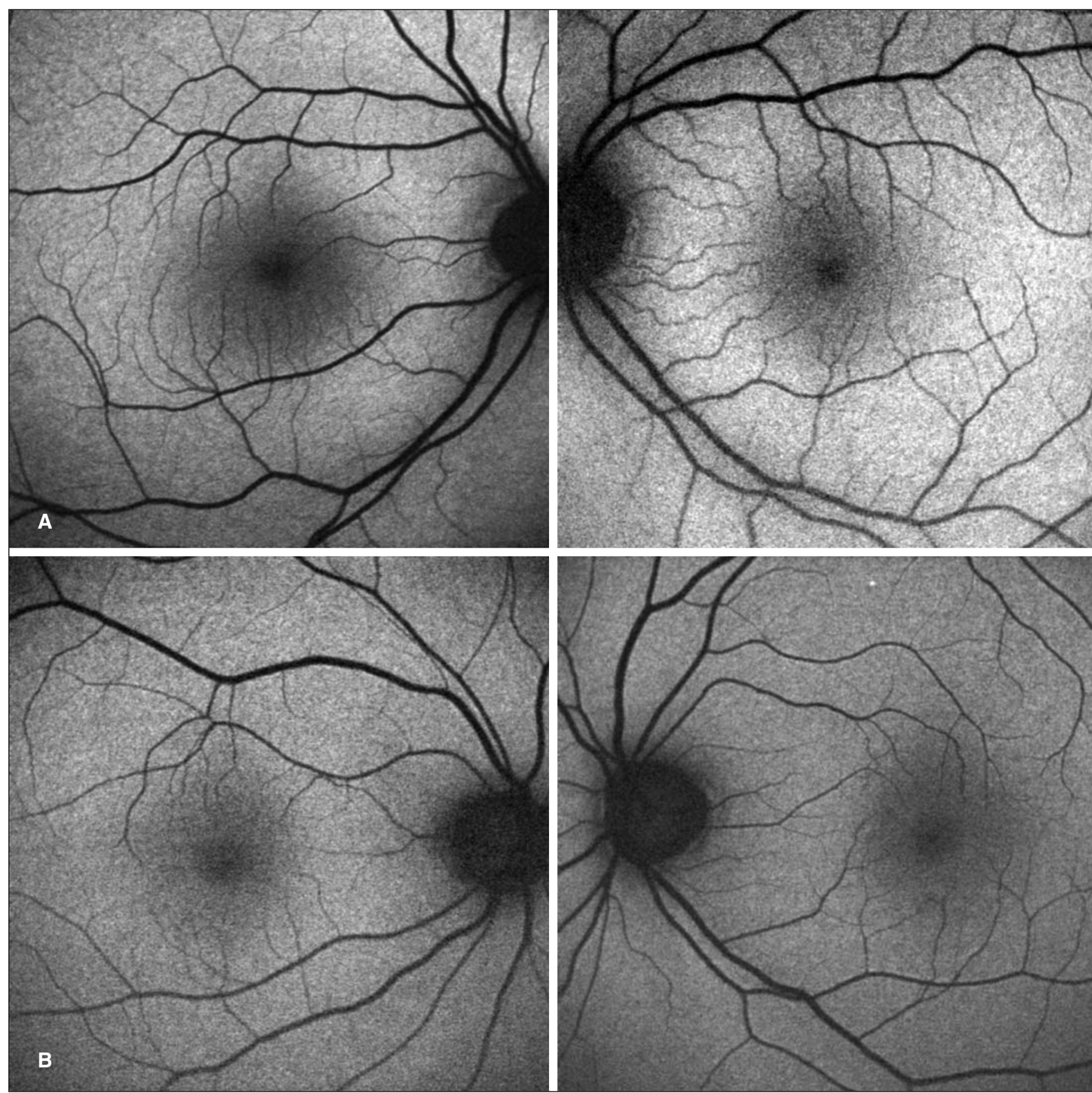

Figura 3 - Imagens autofluorescentes de voluntários saudáveis, sem alterações fundoscópicas. A: 21 anos, sexo masculino, acuidade visual (AV) 20/20,20/20. B: 37 anos, sexo feminino, AV 20/20,20/20. 
imagens. Os pacientes com fotofobia e/ou baixa visão realizaram o exame com maior conforto fixando a luz externa do aparelho. As imagens necessárias para a formação da imagem autofluorescente dos pacientes com nistagmo foram selecionadas manualmente.

\section{Grupo controle}

Ao exame foi claramente observado hipoautofluorescên- cia na região foveal em relação à retina e padrão regular de autofluorescência que será utilizado para comparação com os demais grupos (Figura 2).

\section{Doença de Stargardt}

Nas imagens autofluorescentes desse grupo, em geral a lesão era central oval, com hipoautofluorescência macular. Em pacientes com "fundus flavimaculatus", a área central era
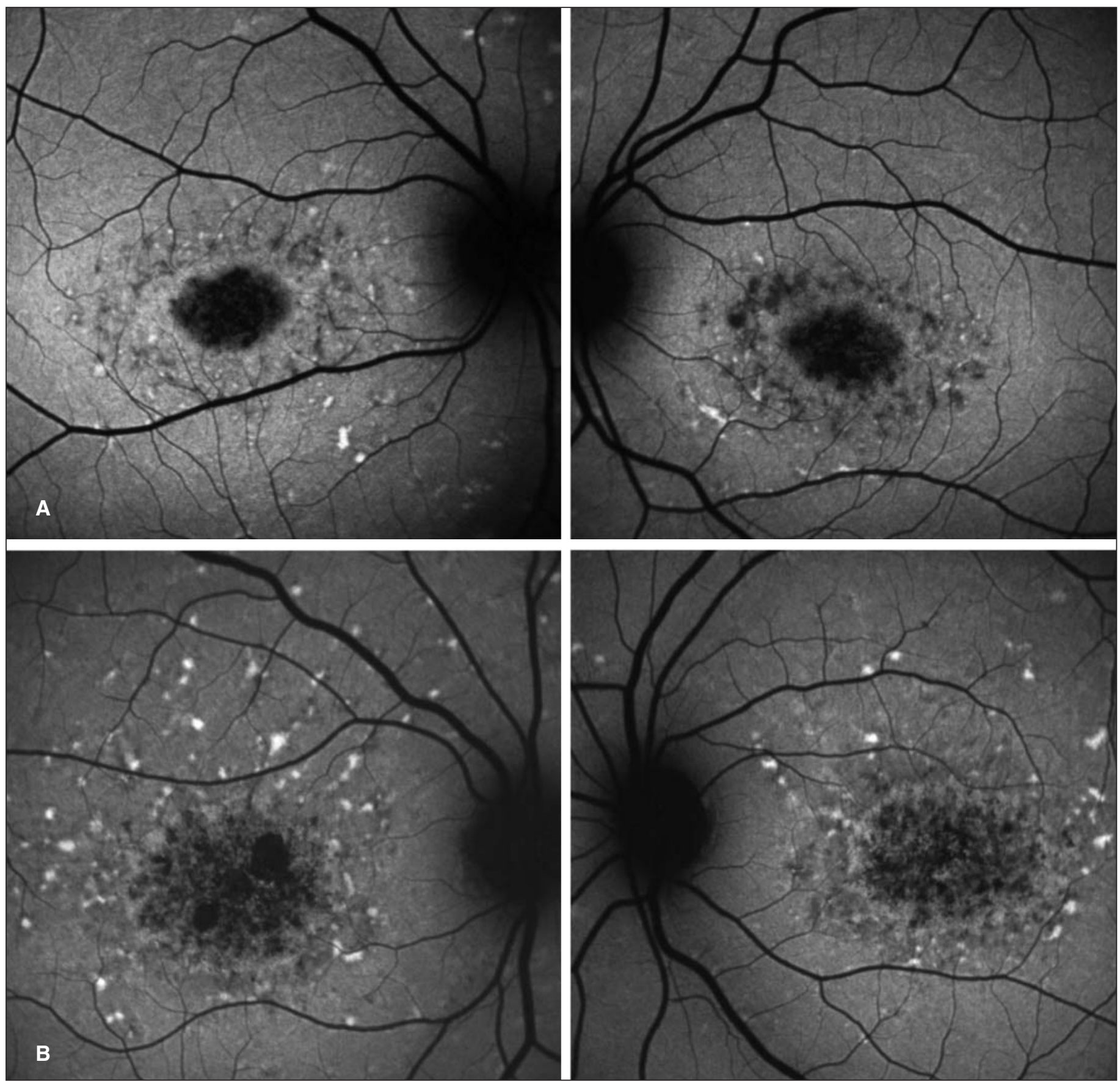

Figura 4 - Autofluorescência em pacientes com doença de Stargardt. A: 24 anos, sexo masculino, AV 20/100,20/100: hipoautofluorescência central com hiper e hipoautofluorescências puntiformes correspondendo a "flecks". B: 37 anos, sexo feminino, AV 20/200,20/200: pontos hipo e hiperautofluorescentes intercalados na área macular. 
rodeada por "flecks" alternando características de hiper e hipoautofluorescências (Figura 3).

\section{Distrofia de cones}

Em seis pacientes com distrofia de cones foi encontrado hipoautofluorescência macular. Em três pacientes, havia halo hiperautofluorescente ao redor da área foveal. E um padrão diferente com desorganização da área macular em apenas um dos pacientes (Figura 4).

\section{Retinose pigmentar}

Em três pacientes, observou-se hipoautofluorescência macular. Em dois, halo hiperautofluorescente ao redor da área foveal. Em quatro, desorganização do padrão de autofluo-
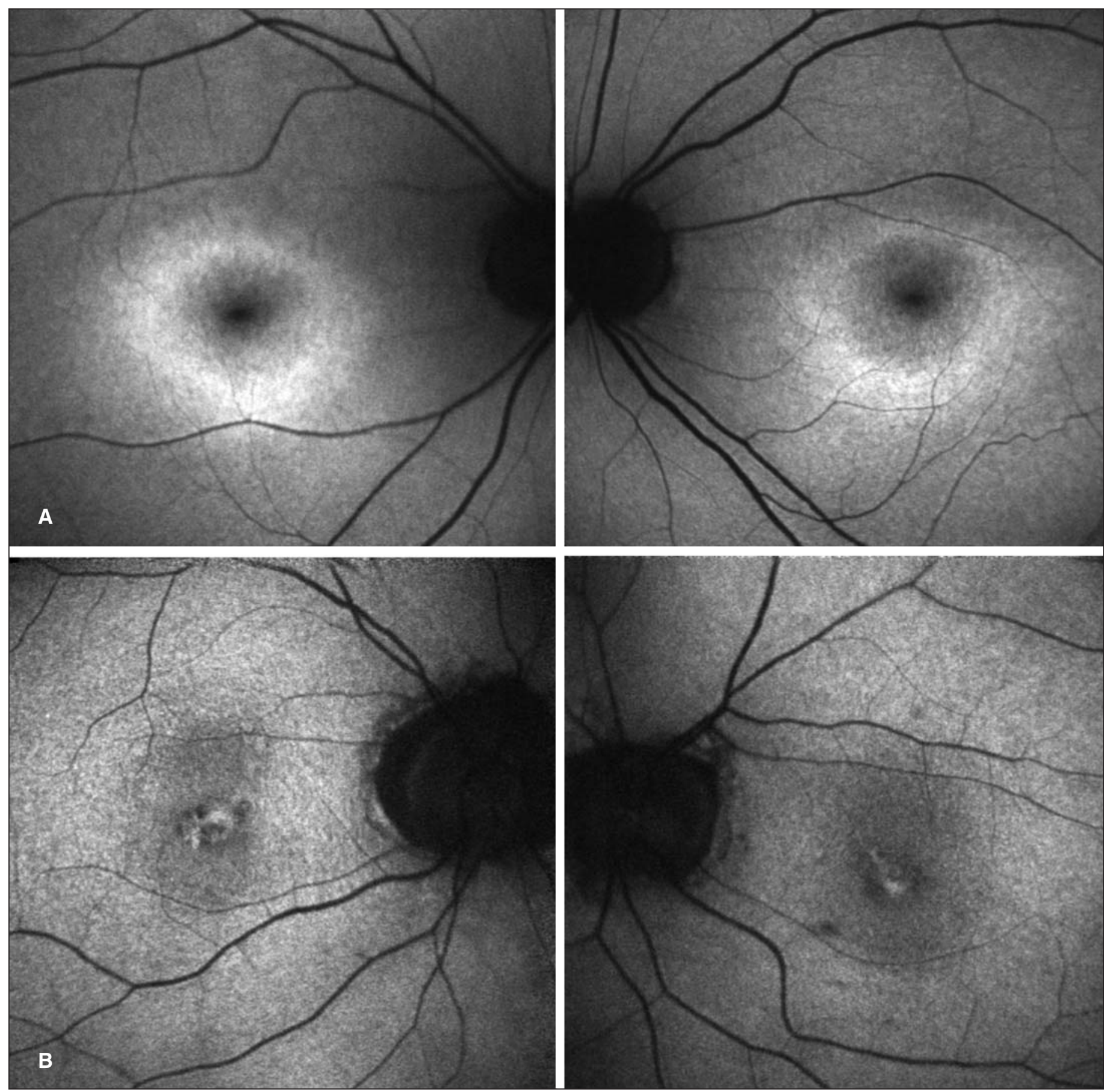

Figura 5 - Autofluorescência em pacientes com distrofia de cones. A: 53 anos, sexo feminino, AV 20/400,20/400: hipoautofluorescência macular com hiperautofluorescência peri macular formando "bull's eye". B: 54 anos, sexo feminino, AV 20/125,20/125: área macular com desorganização do padrão de autofluorescência causando hiper em lesão central rodeado por hipoautofluorescência. 
rescência macular. Já pigmentos periféricos hipoautofluorescentes foram observados em todos os pacientes (Figura 5).

\section{DISCUSSÃO}

Estudos mostram que áreas com aumento de autofluorescência são obtidas através do acúmulo da lipofuscina, e poste- riormente, quando há morte das células fotorreceptoras e conseqüente atrofia, essa área mostra diminuição da autofluorescência, sugerindo que o exame de autofluorescência seja um bom marcador para a integridade do epitélio pigmentar da retina, podendo ser útil para investigar a evolução das distrofias da retina ${ }^{(7)}$.

A alteração do ciclo visual nos casos de distrofias de retina
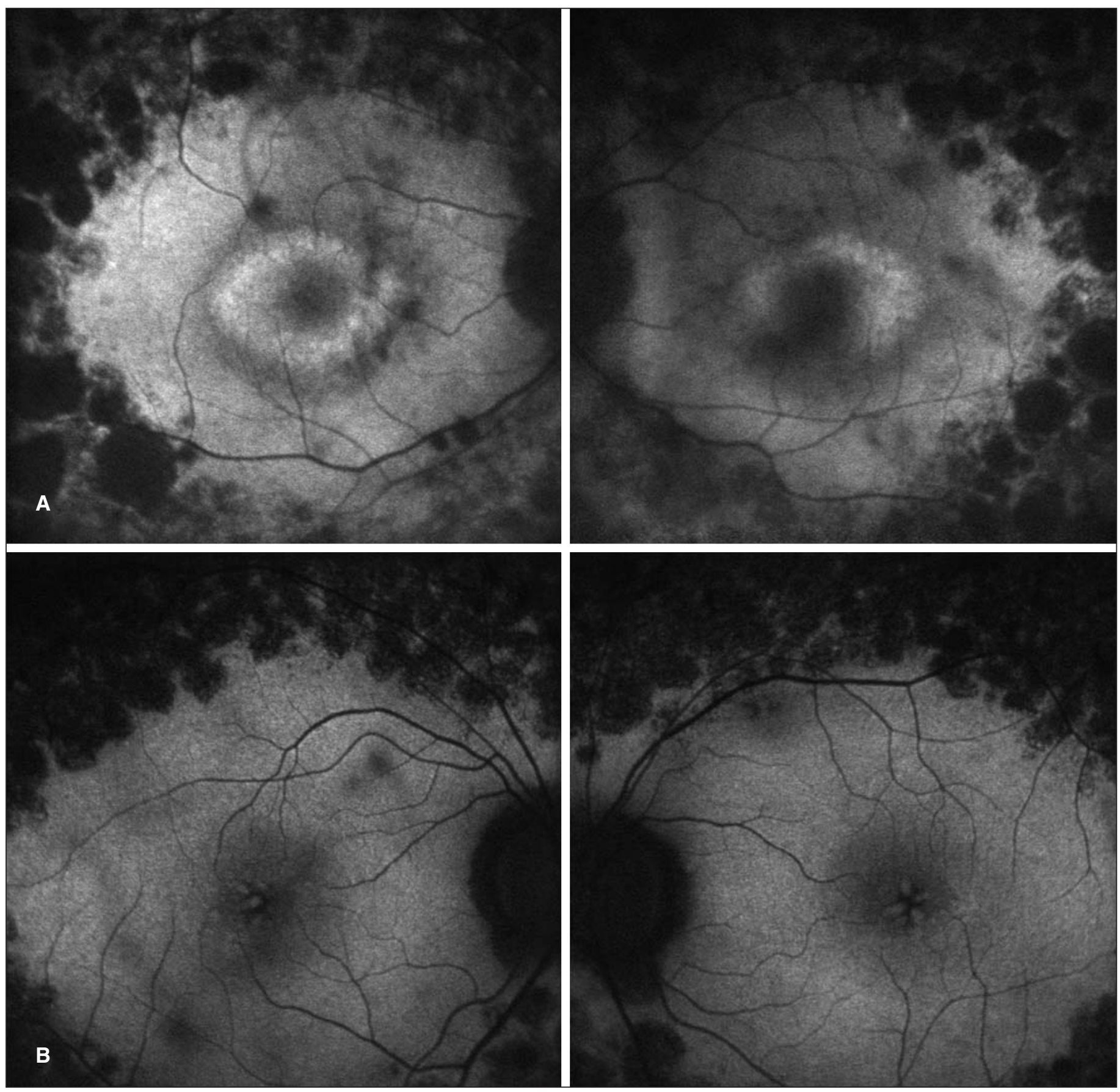

Figura 6 - Autofluorescência de pacientes com retinose pigmentaria. A: 36 anos, sexo feminino, AV 20/200,20/200: hipoautofluorescência central, halo hiper com hipoautofluorescência na margem. B: 38 anos, sexo masculino, AV 20/100,20/100: pigmentos hiper e hipoautofluorescentes na região macular com aspecto de roda de carroça. Hipoautofluorescência nas áreas mais periféricas com deposição de pigmento tanto por atrofia da retina quanto pela presença dos pigmentos depositados. 
causa acúmulo de lipofuscina no EPR e conseqüente morte precoce das células. Clinicamente observa-se atrofia da retina.

A tradução disto é nítida neste estudo. Hiperautofluorescência reflete o depósito de lipofuscina anormal. Quando o processo patológico é tão intenso causando excesso de depósito e atrofia retiniana observa-se hipoautofluorescência.

No grupo de pacientes com distrofias retinianas, principalmente nos grupos dos portadores de distrofia de cones e retinose pigmentar, as lesões não evoluíram da mesma forma. Em alguns pacientes jovens há maior área de atrofia da lesão, do que nos pacientes com mais idade. A intensidade e o padrão de distribuição das lesões que alteram a autofluorescência levam à interpretação de que o acúmulo de lipofuscina precede a atrofia do complexo EPR fotorreceptor.

Através disso, notou-se que a idade não foi um fator de influência significativo na intensidade das alterações fundoscópicas.

Os parâmetros do aparelho foram alterados para conseguir captar imagens melhores de alguns pacientes que apresentaram nistagmo, baixa de visão acentuada $(<1,3 \log$ MAR, 20/ 400 Snellen) e, portanto, não conseguiam fixar adequadamente a mira de fixação, mas nem nessas condições foi possível obter sucesso, por isso suas imagens não foram incluídas na análise.

\section{CONCLUSÃO}

O estudo mostrou a existência de padrões de autofluorescência de fundo nas distrofias de retina que permitem o diagnóstico e melhor interpretação da fisiopatogenia destas doenças.

\section{ABSTRACT}

Purpose: To define characteristics of the fundus autofluorescence examination, verifying usefulness in the diagnosis and care of hereditary retinal diseases. Methods: 28 patients, adults, divided equally into four groups with diagnoses of Stargardt macular dystrophy, cone dystrophy, retinitis pigmentosa and healthy volunteers for the establishment of the normality pattern. An average of nine images with the filter for fluorescein angiography was obtained for the formation of the image autofluorescence using Heidelberg Retina An- giograph2. The images of each group of patients were analyzed to verify common characteristics. Results: The fundus autofluorescence of healthy volunteers showed the foveal area darker than the surrounding retina. The images of Stargardt macular dystrophy, in general, presented an oval central lesion, with reduced autofluorescence. The main alterations of the autofluorescence in patients with cone dystrophy were reduced foveal autofluorescence with a parafoveal ring of increased autofluorescence. In general, the images of retinitis pigmentosa showed outlying pigments with reduced autofluorescence, and of the foveal area, in some cases disorganization or reduced autofluorescence. Conclusion: The study showed the existence of patterns of fundus autofluorescence in the hereditary retinal diseases that allow the diagnosis and better interpretation of the pathogenesis of these diseases.

Keywords: Retinal diseases; Lipofuscin; Pigment epithelium of eye; Eye diseases; Hereditary; Fluorescein angiography

\section{REFERÊNCIAS}

1. Von Rückmann A, Fitzke FW, Bird AC. Distribution of pigment epithelium autofluorescence in retinal disease state recorded in vivo and its change over time. Graefe's Arch Clin Exp Ophthalmol. 1999;237(1):1-9.

2. Bui TV, Han Y, Radu RA, Travis GH, Mata NL. Characterization of native retinal fluorophores involved in biosynthesis of A2E and lipofuscin-associated retinopathies. J Biol Chem. 2006;281(26):18112-9.

3. Sparrow JR, Fishkin N, Zhou J, Cai B, Jang YP, Krane S, et al. A2E, a byproduct of the visual cycle. Vision Res. 2003;43(28):2983-90.

4. Sparrow JR, Boulton M. RPE lipofuscin and its role in retinal pathobiology. Exp eye Res. 2005;80(5):595-606.

5. Cideciyan AV, Swider M, Aleman TS, Sumaroka A, Schwartz SB, Roman MI, et al. ABCA4-Associated Retinal Degenerations Spare Structure and Function of the Human Parapapillary Retina. Invest Ophthalmol Vis Sci. 2005;46(12): 4739-46.

6. Cideciyan AV, Aleman TS, Swider M, Schwartz SB, Steinberg JD, Brucker $\mathrm{AJ}$, et al. Mutations in ABCA4 result in accumulation of lipofuscin before slowing of the retinoid cycle: a reappraisal of the human disease sequence. Hum Mol Genet. 2004;13(5):525-34.

7. Wabbels B, Demmler A, Paunescu K, Wegscheider E, Preising MN, Lorenz B. Fundus autofluorescence in children and teenagers with hereditary retinal diseases. Graefe's Arch Clin Exp Ophthalmol. 2006;244(1):36-45.

8. Schmitz-Valckenberg S, Bültmann S, Dreyhaupt J, Bindewald A, Holz FG, Rohrschneider K. Fundus autofluorescence and fundus perimetry in the junctional zone of geographic atrophy in patients with age-related macular degeneration. Invest Ophthalmol Vis Sci. 2004;45(12):4470-76. Erratum in: Invest Ophthalmol Vis Sci. 2005;46(1):7.

9. Unonius N, Farah ME, Sallum JMF. Classificação diagnóstica dos portadores de doenças degenerativas de retina, integrantes dos grupos Retina São Paulo e Retina Vale do Paraíba. Arq Bras Oftalmol. 2003;66(5):443-8.

10. Bonomo PP, Cunha SL. Doenças da mácula. São Paulo: Roca; 1993. 